\title{
Genescene: Biomedical Text And Data Mining
}

\author{
Gondy Leroy ${ }^{1}$, Hsinchun Chen ${ }^{1}$, Jesse D. Martinez ${ }^{2}$, Shauna Eggers ${ }^{1}$, Ryan R. Falsey ${ }^{2}$, \\ Kerri L. Kislin ${ }^{2}$, Zan Huang ${ }^{1}$, Jiexun $\mathrm{Li}^{1}$, Jie Xu${ }^{1}$, Daniel M. McDonald ${ }^{1}$, Gavin $\mathrm{Ng}^{1}$ \\ Management Information Systems ${ }^{1}$, Arizona Cancer Center ${ }^{2}$ \\ The University of Arizona \\ gleroy@eller.arizona.edu; hchen@eller.arizona.edu; jmartinez@azcc.arizona.edu; \\ seggers@email.arizona.edu; rfalsey@u.arizona.edu; kkislin@u.arizona.edu; \\ zhuang@eller.arizona.edu; jiexun@eller.arizona.edu; jxu@eller.arizona.edu; \\ dmm@eller.arizona.edu; tgavinng@ai.bpa.arizona.edu
}

\begin{abstract}
To access the content of digital texts efficiently, it is necessary to provide more sophisticated access than keyword based searching. Genescene provides biomedical researchers with research findings and background relations automatically extracted from text and experimental data. These provide a more detailed overview of the information available. The extracted relations were evaluated by qualified researchers and are precise. A qualitative ongoing evaluation of the current online interface indicates that this method to search the literature is more useful and efficient than keyword based searching.
\end{abstract}

\section{Introduction}

The Internet has increased the availability of and access to publications, leading in many cases to information overload. In biomedicine, this effect has been accelerated by an increase in publications and datasets due to the decoding of the human genome. Every 11 years, the number of researchers doubles [6] and Medline, the main resource of research literature, has been growing with more than 10,000 abstracts per week since 2002 [5]. In addition, large amounts of gene expression data involving expression measurements of thousands of genes, are available from microarray experiments. Gene expression patterns embedded in the data can potentially lead to the discovery of unknown genetic relations.

The information overload problem for both literature and data analysis calls for solutions that can largely automate these processes. Genescene, a toolkit developed for biomedicine, will provide more adequate access to text and data. It will allow researchers to view the findings extracted from Medline abstracts, and compare them with findings from microarray experiments.

\section{Related Work}

To access the content of documents, natural language processing (NLP) is required. Existing techniques range from simple pattern matching to full parsers. In biomedicine, many NLP approaches start from a list with specific gene names or verbs and extract the surrounding text as relations $[2 ; 7 ; 8]$. The best among these achieve high precision but low recall since few relations are extracted. More general co-occurrence based approaches assume that phrases, e.g., genes, are related when they appear together in a text [3]. This approach extracts more relations but they are less precise. Both methods ignore negation.

\section{Genescene}

Genescene combines relations between entities in text extracted by a rule-based parser and a corpus-based cooccurrence analysis technique. It will also extract regulatory relations from microarray data and combine these with the relations extracted from text.

\subsection{Rule-based findings}

The rule-based parser, successfully tested with a small prototype [4], is based on closed-class words which provide a generic structure for the relations. Cascaded finite state automata (FSA), built around prepositions and basic sentence elements, identify the structures. Each FSA incorporates negation, an important element ignored by others, and also captures relations based on conjunctions. For example, from the sentence "Thus hsp90 does not inhibit receptor function solely by...," the relation "NOT: hsp90 - inhibit - receptor function" is extracted. 


\subsection{Corpus-based background}

The corpus-based background relations represent the knowledge in the entire domain and form the background for the rule-based relations. These corpus-based relations are formed by a co-occurrence-based algorithm tested earlier in an information retrieval context [1]. They represent relations between noun phrases that hold true for the entire collection.

\subsection{Ontologies}

Three ontologies, the Gene Ontology (GO), the Human Genome (HUGO) Nomenclature, and the Unified Medical Language System (UMLS), are used to better integrate the relations. A term can receive multiple semantic tags based on its being in the ontologies.

\subsection{Regulatory relations from data}

Regulatory relations are extracted from microarray data using data mining techniques such as Bayesian networks and association rule mining. Incorporating these regulatory relations into Genescene will help researchers compare experimental discovery with previous knowledge from literature. Unexpected gene associations can be identified to guide further literature search or new experimental design.

\subsection{Online Access}

Users can currently access Genescene's text mining demo (http://ai.bpa.arizona.edu/go/genescene). Keywords are used to retrieve relations found in the Medline abstracts and users can choose the type of relation they want to see. A list of relations is then shown for the search, ordered by the type and number of elements in the relation. The number of abstracts containing the relations is also shown. Clicking on a relation retrieves the list of associated abstracts. The relations and search terms are highlighted in the abstract text.

\section{Case study}

The extracted relations and online interface are being evaluated with quantitative and qualitative studies.

\subsection{Genescene relations}

Two researchers evaluated the rule- and corpus-based relations from $\mathrm{p} 53$-related abstracts. The rule-based relations were $95 \%$ correct and the corpus-based relations were $60 \%$ correct. Limiting the corpus-based relations to those that had entities with ontology tags increased their correctness to $78 \%$. Most terms and relations were considered relevant, especially when part of an ontology.

\subsection{Genescene content}

Encouraged by the excellent evaluation results, three collections with biomedical abstracts were added to Genescene. Different research groups requested the collections. Table 1 provides an overview. The P53 collection contains all abstracts available in Medline (up to and including summer 2002) with the keyword "p53" in the title or abstract. The AP1 collection is similarly based on the keywords: ap1, ap-1, jnk, erk, jun, fos, and p38. The yeast collection is based on the keyword "yeast." Hundreds of thousands of relations are available for each collection. In each, more than half of the terms received a tag from an ontology. The UMLS provided more than $50 \%$ of the terms with a tag. Slightly more than $1 \%$ of the terms received a GO-tag. HUGO provided the least tags, except for the yeast collection where $1.4 \%$ of the terms received a HUGO tag.

Table 1. Overview of Genescene's content

\begin{tabular}{|rccc|}
\hline Topic: & P53 & AP1 & Yeast \\
\hline Abstracts: & 23,234 & 30,820 & 56,246 \\
\hline Rule-B. Relations: & 270,008 & 387,666 & 560,165 \\
Corpus-B. Relations: & $5,023,103$ & $6,526,454$ & $7,736,647$ \\
\hline Terms w. UMLS tag: & $57 \%$ & $54 \%$ & $51 \%$ \\
Terms w. HUGO tag: & $0.6 \%$ & $0.9 \%$ & $1.4 \%$ \\
Terms w. GO tag: & $1.1 \%$ & $1.1 \%$ & $1.1 \%$ \\
\cline { 2 - 4 } Any ontology tag: & $58 \%$ & $55 \%$ & $52 \%$ \\
\hline
\end{tabular}

\subsection{Genescene interface}

The ordering of the relations was very much liked. Researchers felt the most important relations, e.g., conclusions, were presented first. They also liked the highlighting of relations and keywords in the original abstracts. However, currently only one keyword or phrase can be used to search a collection. A multiple-keyword search should be added and the speed of partial match queries for background relations should be improved.

\section{Future Directions}

In the future, we will integrate both the rule- and corpus-based relations in an interactive graphical map display. Later, visual text mining will become possible. For example, users will be able to search for specific time ranges in the publications or for specific organism. Furthermore, findings from text will be incorporated into microarray data mining results to improve the algorithmic 
performance and vice versa microarray findings will lead to automatic literature searches.

\section{Acknowledgements}

Genescene is supported by the grant: NIH/NLM, 1 R33 LM07299-01, 2002-2005, “Genescene: a Toolkit for Gene Pathway Analysis." We thank the National Library of Medicine, the Gene Ontology Consortium, and the Hugo Nomenclature Committee for making the ontologies available to researchers.

\section{References}

[1] Chen, H. and Lynch, K. J. Automatic Construction of Networks of Concepts Characterizing Document Databases. IEEE Transactions on Systems, Man and Cybernetics, 22, 5 (1992), 885-902.

[2] Friedman, C., Kra, P., Yu, H., Krauthammer, M. and Rzhetsky, A. GENIES: a natural-language processing system for the extraction of molecular pathways from journal articles. Bioinformatics, 17, Suppl. 1 (2001), S74-S82.
[3] Jenssen, T.-K., Laegreid, A., Komorowski, J. and Hovig, E. A literature network of human genes for highthroughput analysis of gene expression. Nature Genetics, 28, (2001), 21-28.

[4] Leroy, G. and Chen, H. Filling Preposition-based Templates to Capture Information from Medical Abstracts. Paper presented at the Pacific Symposium on Biocomputing, (2002), 350-361.

[5] National-Library-of-Medicine, Fact Sheet - Medline. National Library of Medicine: http://www.nlm.nih.gov/pubs/factsheets/medline.html

[6] Perutz, M. F. Will biomedicine outgrow support? Nature, 399, (1999), 299-301.

[7] Pustejovsky, J., Castaño, J., Zhang, J., Kotecki, M. and Cochran, B. Robust Relational Parsing Over Biomedical Literature: Extracting Inhibit Relations. Paper presented at the Pacific Symposium on Biocomputing (2002), 362-373.

[8] Thomas, J., Milward, D., Ouzounis, C., Pulman, S. and Carroll, M. Automatic Extraction of Protein Interactions from Scientific Abstracts. Paper presented at the Pacific Symposium on Biocomputing (2000), 538-549. 